\title{
Exercise during Pregnancy: Developmental Programming Effects and Future Directions in Humans
}

\author{
Authors \\ Polina M. Krassovskaia1, 2, Alec B. Chaves, 2, Joseph A. Houmard1,2, Nicholas T. Broskey1,2
}

\section{Affiliation}

1 Human Performance Laboratory, Department of Kinesiology, East Carolina University, Greenville, United States

2 East Carolina Diabetes \& Obesity Institute, East Carolina University, Greenville, United States

Key words developmental programming, exercise, pregnancy, mitochondria

accepted 27.05.2021

published online $\quad 03.08 .2021$

\section{Bibliography}

Int J Sports Med 2022; 43: 107-118

DOI 10.1055/a-1524-2278

ISSN 0172-4622

(C) 2021. Thieme. All rights reserved. Georg Thieme Verlag KG, Rüdigerstraße 14, 70469 Stuttgart, Germany

\section{Correspondence}

Dr. Nicholas T. Broskey

Kinesiology, East Carolina University

115 Heart Drive

27834 Greenville

United States

Tel.: + 2527374684 , Fax: + 2527374684

broskeyn19@ecu.edu

\begin{abstract}
Epidemiological studies show that low birth weight is associated with mortality from cardiovascular disease in adulthood, indicating that chronic diseases could be influenced by hormonal or metabolic insults encountered in utero. This concept, now known as the Developmental Origins of Health and Disease hypothesis, postulates that the intrauterine environment may alter the structure and function of the organs of the fetus as well as the expression of genes that impart an increased vulnerability to chronic diseases later in life. Lifestyle interventions initiated during the prenatal period are crucial as there is the potential to attenuate progression towards chronic diseases. However, how lifestyle interventions such as physical activity directly affect human offspring metabolism and the potential mechanisms involved in regulating metabolic balance at the cellular level are not known. The purpose of this review is to highlight the effects of exercise during pregnancy on offspring metabolic health and emphasize gaps in the current human literature and suggestions for future research.
\end{abstract}

\section{Introduction}

It is well established that a sedentary lifestyle is associated with an increased incidence of chronic diseases, such as type 2 diabetes, cancers, cardiovascular diseases, and comorbidities [1]. To reduce this burden, effective interventions need to be discerned and implemented. Physical activity is an accessible positive lifestyle habit that can contribute to weight loss, changes in body composition, and improved cardiorespiratory fitness [2-9]. Physical activity interventions have shown to be successful in increasing an individual's quality of life $[10,11]$ and continue to support the need for physical activity across all races, ethnicities, genders, and age groups. Once thought to be detrimental to the developing fetus, exercise is now recommended for most pregnant women [12]. The notion of increasing physical activity interventions in pregnancy is gaining traction in that this period is a crucial timepoint to improve offspring outcomes.

In 1989, the epidemiologist David Barker coined the Developmental Origins of Health and Disease Hypothesis by linking small for gestational age infants with increased incidence of cardiovascular disease in adulthood [13]. This hypothesis postulates that if the fetus is exposed to unfavorable environmental conditions in utero and during the early stages of development, the fetus will undergo permanent metabolic adaptations that allow for survival in the unfavorable intrauterine environment. However, these adaptations may also lead to the development of diseases after birth [14]. Excess maternal weight gain and obesity during pregnancy have been known to contribute to poor fetal and maternal out- 
comes related to risk factors/incidence for cardiometabolic disease [15] making pregnancy a potentially viable target for intervention. The cyclic nature of cardiometabolic diseases continuing from mother to child and the subsequent perpetuation of metabolic disease across generations marks a potentially dire need for interventions to halt this vicious cycle. Luckily, pregnancy has been identified to have the potential to be a "teachable moment" for mothers [16] due to the increased contact with healthcare providers and increased concern for the health of the fetus. The purpose of this review is to provide a synopsis on physical activity as a method of improving neonatal metabolic health. This review will begin with general guidelines on physical activity during pregnancy and a brief section on maternal responses. It will then turn its attention toward offspring responses to maternal physical activity that encompass changes in whole-body and cellular metabolism. Finally, potential areas of investigation for future research will be presented.

\section{Exercise During Pregnancy}

\section{Physical activity recommendations}

Pregnant women can benefit from physical activity to a similar extent as nonpregnant women [17], and various forms of physical activity have been deemed safe and appropriate during pregnancy [12, 17-19]. The American College of Obstetricians and Gynecologists (ACOG) currently recommends that women who begin their pregnancy with a "healthy lifestyle" (e.g., exercise, proper nutrition, nonsmoking) continue to maintain those healthy habits throughout their pregnancy [12]. Women not achieving "healthy lifestyle" habits should accordingly be encouraged to establish healthier habits and routines throughout the pre-pregnancy and pregnancy periods [12]. During pregnancy, 150 minutes of moderate intensity aerobic activity per week is recommended [12, 19]. Those who habitually engaged in vigorous intensity aerobic activity or who were physically active before pregnancy can continue their activities [12]. Consistent with recommendations from the American College of Sports Medicine (ACSM) [20], a combination of aerobic and resistance exercise appears to deliver benefits for both the mother and infant. This review will highlight beneficial changes with primarily aerobic exercise/physical activity.

For women with uncomplicated pregnancies, fears of physical activity and exercise resulting in adverse outcomes have yet to be validated [21-24]. While these exercise recommendations have been in place for over a decade, the prevalence of active pregnant women is still alarmingly low. Among pregnant women, walking is the most frequently reported activity, usually occurring during the first trimester $[25,26]$. Across the United States however, it is estimated that as few as $15.8 \%$ of women are physically active at the recommended level during pregnancy [27]. Only $21.5 \%$ of a cohort of healthy pregnant women in Ireland reported meeting the current ACOG recommendations of physical activity with $11.7 \%$ reporting no physical activity at all [28]. Studies across other countries report similar numbers [29-31]. Further, for those who do participate in structured physical activity during pregnancy, the intensity, frequency, and volume may not be at levels sufficient to incur the adaptations induced with an active lifestyle [32]. Aerobic exercise interventions have been found to minimize gestational weight gain when combined with diet or with exercise alone [33]. However, a recent multi-site randomized clinical trial that managed to increase physical activity showed a modest effect between the intervention and control group $(-1.59 \mathrm{~kg})$ on total gestational weight gain [34] and did not prevent gestational diabetes in the mother [35]. Nonetheless, exercise has been shown to be protective against disorders such as preeclampsia and should be promoted due to several beneficial physiological adaptations [36-40].

\section{Maternal responses during pregnancy}

The scope of this article is offspring outcomes in response to maternal exercise; therefore, this review will briefly touch upon maternal adaptations. It should be noted that the effects of exercise on pregnant women has been reviewed extensively elsewhere [4143]. During a healthy pregnancy, many physiological adaptations occur in the cardiovascular system to support adequate oxygen and nutrient supply to the fetus. Cardiac output is increased up until term by 30 - to 50 percent due to both an increase in stroke volume and heart rate (HR) [44]. An additional increase in tidal volume is responsible for a 30 - to 40 percent increase in minute ventilation in pregnancy. Although many of these changes would assume a rise in oxygen consumption, there is only a slight 15- to 20 percent increase, resulting in an increase in alveolar and arterial $\mathrm{PaO}_{2}$ (partial pressure of oxygen) and a fall in $\mathrm{PaCO}_{2}$ (partial pressure of carbon dioxide) levels [45]. These and other positive adaptations that occur with pregnancy are amplified with regular physical activity and exercise. Cardiovascular fitness, measured by maximal oxygen uptake $\left(\mathrm{VO}_{2} \mathrm{max}\right)$, is rarely reported with pregnancy due to theoretical risk of fetal distress. However, there are instances where this has been performed in pregnancy [46]. As central responses (e. g. stroke volume, HR, cardiac output, etc.) do not differ significantly between pregnant and nonpregnant women during submaximal exercise [47], it seems that alterations in the periphery are at play.

Many of the peripheral cardiovascular changes seen in physically active mothers help to ensure the appropriate trafficking of nutrients to the developing fetus. Because the placenta is the central organ linking the fetus and the maternal environment, it is responsible for bridging the effects of external stimuli on maternal health status to the fetus. Placental growth is largely dictated through substrate availability and blood flow and is calculated as the product of substrate concentration measured in arterial blood and blood delivery to the placental bed, with a heavy focus on glucose [4850]. With maternal exercise, blood flow is diverted from the placenta to exercising muscles and skin [51] which is proportional to the exercise intensity and muscle mass used [48]. After the cessation of exercise, blood flow quickly returns to normal [48]. Due to the invasive nature of measuring fetoplacental blood flow, exerciseinduced blood redistribution has not been measured in humans. Animal data translated to humans, however, indicates blood flow redistribution associated with exercise intensities up to $95 \%$ $\mathrm{VO}_{2}$ max does not compromise the fetus. Repeated bouts of exercise at $95-100 \% \mathrm{VO}_{2} \mathrm{max}$, however, are associated with negative effects on fetal growth confirming submaximal exercise does not compromise blood delivery to the fetus [43, 52-54].

Additionally, maternal exercise impacts placental gene expression to optimize fetal nutrient delivery and fetoplacental growth [55-57]. Those who performed strenuous exercise during preg- 
nancy had increased T-type amino acid transporter 1 (TAT1), neutral amino acid transporter $A(A S C T 1)$, mitochondrial branched chain amino transferase ( $m B C A T)$, and glutamine sythetase (GLUL) placental expression indicating maternal exercise enhances amino acid transport pathways [58]. Genes associated with fatty acid metabolism are similarly altered with maternal exercise [59-61]. Mothers who met physical activity guidelines also showed improvements in the expression of genes involved in glucose transport as well as mammalian target of rapamycin (mTOR) and insulin signaling in the placenta, further highlighting the benefits of maternal exercise in the relationship between the maternal environment, placenta, and fetal environment [55]. Finally, reactive oxygen species (ROS) production in the placenta was also lowered with exercise suggesting improved oxygen metabolism [57]. All these beneficial adaptations are imparted in the offspring to ensure adequate growth.

\section{Effects of Maternal Exercise on Offspring}

\section{Anthropometrics}

Infant birth weight allows for a crude measurement of newborn health and is an indicator of the fetal environment. Both low and high birth weights have been shown to be related to obesity, metabolic disease, and cardiovascular disease later in life [62-64]. The pregnancy field has outlined a clear U-shaped association between offspring birth weight and long-term metabolic complications [65]. Many of these have been outlined in epidemiological studies. For example, studies on famine in pregnancy concluded that infants exposed to conditions of malnutrition have reductions in glucose tolerance later in life [66]. In the case of maternal obesity, infants have increases in childhood body mass index (BMI), adiposity, and increased risk of diabetes as adults $[67,68]$. Therefore, there is a need to fine tune this $U$-shaped association with lifestyle interventions, with one of the most prominent interventions being exercise.

Largely conflicting evidence exists for the support of structured maternal exercise affecting infant birth weight. Maternal exercise during pregnancy has been associated with increased infant lean mass compared to infants of sedentary mothers [69]. Other studies have shown that maternal exercise has been shown to be associated with a reduction in the upper quantiles of birth weight distributions $[70,71]$. There are reports showing that maternal exercise may not affect infant weight at birth [72]; however, a recent study has shown that infants exposed to maternal exercise had increased adiposity at 7-years of age [69]. Finally, a recent meta-analysis conducted by Guillemette et al. concluded prenatal maternal exercise does not significantly impact infant birth weight nor fat mass nor large-for-gestational-age risk [73]. These studies highlight the continued need for more studies focused on maternal exercise and infant birth weight. Indeed, studies of regular aerobic exercisers and those who engage in vigorous physical activities, such as elite athletes, show that infants were born with lower birth weight $[74,75]$. Thus, there might be a dose-response relationship between maternal exercise, again lending credence to the finetuned nature of maternal pregnancy outcomes and the U-shape association that also exists in other aspects of pregnancy such as that in gestational weight gain [76].

Research on other forms of physical activity, such as non-structured leisure time physical activity (LTPA) has also been studied. Research has shown that LTPA does not increase the chance of a small for gestational age newborn [77]. At a minimum, adherence to physical activity guidelines has been shown to reduce risk of delivering large for gestational age newborn with no effect on delivering small for gestational age [71, 78-80]. LTPA is thought to normalize birth weight into a healthy range by normalizing maternal blood glucose, reducing maternal insulin resistance, and altering placental blood flow and nutrient delivery [32, 49, 81, 82]. While remaining cautious to not over-interpret these results, enough evidence of lasting benefits of LTPA during pregnancy exists to encourage larger, prospective studies to understand if prenatal interventions might be an effective way of preventing childhood obesity in humans.

With limited data on long-term outcomes of offspring to exercising mothers in humans, rodent studies may provide additional insights. In mice, maternal exercise improves offspring body composition $[83,84]$ or shows no effect $[72,85]$. Interestingly, when fed a high-fat diet, offspring of trained mothers gained less weight and stored less fat compared to offspring of untrained mothers, which suggests a protective effect of exercise [72, 83]. While rodent findings may not be applicable in human research, due to factors such as uterine structure, length of gestation, size of litters, the idea of exercise as a protective measure to support a more favorable body composition is persuading.

\section{Cardiovascular fitness}

Aerobic fitness $\left(\mathrm{VO}_{2} \mathrm{max}\right)$ is the product of central cardiac output and the peripheral oxygen extractability of the working tissues [86]. Exercise training in non-pregnant cohorts increases aerobic fitness via coordinated adaptations of these central and peripheral components and the majority of work in this area has been substantiated in the animal literature. Prior data has shown that rodent offspring born to mothers who underwent aerobic exercise before and during pregnancy, have higher aerobic fitness [87] and physical activity levels [88] providing evidence that maternal exercise is capable of programming the offspring's cardiovascular system including cardiac output, macrovascular compliance, and skeletal muscle oxidative capacity. These are summarized in the subsequent paragraphs.

A hallmark adaptation of chronic exercise training is an increase in stroke volume and increased heart rate variability [89-93]. Although the effect of maternal exercise on offspring cardiovascular remodeling is not yet fully understood, exercise training has been shown to improve ejection fraction and left ventricular mass [94]. Exercise training also prevents obesity-induced impairments in cardiac output by attenuating pathological left ventricular hypertrophy and preserving the ejection fraction of adult rodent offspring [95]. While the mechanisms are not clear, studies have shown that maternal exercise epigenetically programs the offspring's cardiac transcriptome, increasing the expression and activity of genes involved in mitochondrial biogenesis [94, 96] - an important characteristic for many offspring peripheral adaptations. 
Although indirect, aerobic exercise generally improves cardiac function by decreasing afterload via structural and functional changes that increase vascular compliance [97-99]. Maternal exercise has been shown to improve endothelium-dependent vasodilation in porcine offspring at birth, but this effect was blunted in the presence of $\mathrm{N}^{\mathrm{G}}$-nitro-L-arginine methyl ester (L-NAME), a nitric oxide synthase inhibitor, suggesting increased nitric oxide bioavailability is responsible for this improvement [100]. However, followup studies indicated the effects of maternal exercise on offspring endothelium are transient and no longer evident in the months following birth in either healthy [101] or high-fat fed swine offspring [102]. Furthermore, endothelium-independent relaxation in the adult offspring exposed to maternal exercise was reduced, suggesting maternal exercise may accelerate the age-related decline in smooth muscle compliance [101]. In rodents, maternal exercise did not alter the offspring's endothelial-dependent or -independent relaxation in the months following delivery [103]. Similarly, Boonpattrawong et al. reported that maternal exercise alone had no effect on offspring vascular function but was protective against maternal obesity and high fat post-weaning diet. Interestingly, aerobic exercise improved nitric oxide bioavailability in the offspring fed the Western diet, despite the groups having similar levels of nitric oxide synthase expression. The authors determined maternal exercise improved aortic one-carbon metabolism, which could have indirectly improved nitric oxide bioavailability by reducing the uncoupling of nitric oxide synthase in the mice fed the high fat diet [104]. Finally, Li et al., observed that maternal exercise reduced smooth muscle vasoconstriction responses to norepinephrine and Bay K8644 (a Ca ${ }^{2+}$ channel agonist) of offspring born to spontaneous hypertensive mother rats. DNA bisulfite sequencing revealed maternal exercise increased methylation of the calcium voltagegated channel subunit $\alpha-1 C$ (Cacna1c) promoter, preventing it from being upregulated during the programmed hypertension [105]. Taken together, these data suggest maternal exercise can serve a protective role in vascular function, but this protection is only evident with gestational obesity and/or postnatal insults (e. g., highfat diet).

Despite extensive work being conducted in animal models, it is still unclear if these results can be translated to humans. Limited data stems from a recent pilot study revealing moderate-intensity aerobic exercise in healthy pregnant women reduced the carotid intima-media thickness in offspring, suggesting improved vascular compliance [106]. Furthermore, there have been few studies to track the effectiveness of maternal exercise on cardiac function in humans. May et al. determined maternal aerobic exercise reduces fetal heart rate and increases heart rate variability at 36 weeks of gestation [107]. Follow-up analysis by this group revealed the increase in heart rate variability was retained one month following delivery, providing evidence of a lasting cardiovascular phenotype [108]. Whether or not these other mechanisms, such as the nitric oxide system, are at play in humans remains to be discovered.

\section{Skeletal muscle oxidative capacity}

The peripheral component, oxygen extractability $\left(\mathrm{A}-\mathrm{VO}_{2}\right)$, of working skeletal muscle increases with aerobic training via increases in capillary density and mitochondrial biogenesis [109-111]. Like cardiovascular changes, most of this work has been shown using ani- mal models. Liu et al. was the first to establish a programming effect of maternal exercise on the oxidative capacity of rodent skeletal muscle. They and others [87] noted that exercise prior to and during pregnancy did not alter skeletal muscle capillary density but did increase markers of mitochondrial biogenesis including mitochondrial density and the enzymatic activity of citrate synthase and cytochrome $C$ oxidase in the offspring [112]. Further, Siti et al. reported that maternal exercise in rodents increased the enzymatic activity of electron transport system complexes II and III, reduced substrate-specific $\mathrm{H}_{2} \mathrm{O}_{2}$ production, and increased ADP-stimulated respiration rates in offspring skeletal muscle [113]. Peroxisomal proliferator-activated receptor $y$ coactivator- 1 alpha $(P G C-1 \alpha)$ has been termed the "master regulator" of mitochondrial biogenesis and plays a key role in several exercise-induced adaptations [114117]. Therefore, it can be proposed that maternal exercise could epigenetically modify the PGC-1 $\alpha$ gene (Ppargc 1a), to 'prime' PGC$1 \alpha$ expression in the offspring skeletal muscle. Son et. al provided the first evidence that exercise alone reduced the methylation status of the offspring's skeletal muscle Ppargc1a promoter, increasing the expression of PGC- $1 \alpha$. Importantly, the authors noted several other markers of mitochondrial biogenesis and oxidative capacity increased, including increased $\mathrm{VO}_{2} \mathrm{max}$, proportion of oxidative muscle fiber (higher Ila/lower Ilx), mitochondrial DNA (mtDNA) content, and markers of mitochondrial fission/fusion [87]. Taken together, there is accumulating evidence in rodents to suggest maternal exercise enhances the oxidative capacity of the skeletal muscle of offspring via intrinsic changes in the mitochondrial phenotype. However, due to the invasive and longitudinal nature of these studies, these results have yet to be substantiated in humans.

\section{Substrate metabolism}

The increased prevalence of sedentary lifestyles and Western-style diets has led to a parallel rise in metabolic diseases including type 2 diabetes, metabolic syndrome, and cardiovascular disease. A distinctive feature of these diseases is disordered substrate metabolism and the eventual ectopic deposition of substrates and excessive spillover of metabolites [118]. These aspects have significant relevance to the pregnancy field as well [119]. Generally, exercise interventions that aim to improve aspects of substrate handling is concomitant with enhancements in mitochondrial content/function to resolve the perturbations in metabolic stress. Due to the robust changes in the skeletal muscle mitochondrial phenotype elicited by maternal exercise as described in the section above, there is a vested interest in determining whether it can protect the offspring from metabolic dysfunction and disease in the current obesogenic environment.

Thus far, several investigations have determined maternal exercise can reduce the offspring's susceptibility to metabolic diseases by rescuing glucose intolerance, hyperlipidemia, endocrine dysregulation, and global oxidative stress in offspring born to mothers with obesity or that were fed a high-fat diet during pregnancy $[115,120-125]$. These have been shown in models of rodent exercise where mice were trained preconception and in combination of preconception and during pregnancy. Data suggests maternal exercise acts on tissues responsible for regulating whole-body metabolism including the skeletal muscle, liver, and pancreas. 
The skeletal muscle is responsible for $70-80 \%$ of postprandial glucose disposal, thus development of skeletal muscle insulin resistance is a key tenant in the pathogenesis of type 2 diabetes [126]. Carter et al. was the first to show maternal exercise improves ex vivo glucose uptake in the skeletal muscle, but not adipose tissue of the rat offspring [127]. Although the exact mechanisms have yet to be elucidated, data suggests maternal exercise relieves skeletal muscle Ppargc1a promoter hypermethylation, induced by maternal high-fat diet, which was associated with elevations in the mRNA expression of glucose transporter 4 (Glut4), cytochrome c (Cyt c), and cytochrome c oxidase subunit 4 (Cox4)[115]. Furthermore, a recent study indicated maternal exercise protects the offspring's oxidative capacity by rescuing their mitochondria phenotype and fiber type distribution. The authors determined maternal exercise was responsible for demethylating the Ppargc1a promoter and increasing PGC- $1 \alpha$ expression, in contrast to the repression evident with a maternal sedentary lifestyle and high-fat gestational diet [87]. Taken together, maternal exercise improves the substrate handling of the offspring skeletal muscle and offers protection from certain disruptions associated with maternal obesity and Westernstyle gestational diets. However, Quiclet et. al found that maternal exercise did not rescue the glucose tolerance in rat offspring fed a high fat/high sucrose diet. In situ mitochondrial respiration assays revealed maternal exercise improved substrate affinity $\left(K_{m}\right)$ for palmitoyl-CoA and pyruvate in sedentary, chow-fed offspring, but not in mice fed a high-fat/high-sucrose diet [83]. Therefore, it is still unclear if maternal exercise can protect the offspring skeletal muscle from postnatal dietary insults. Moreover, no investigations have been done to determine if these results can be translated to humans.

In coordination with the skeletal muscle, liver metabolism and pancreatic $\beta$-cell function plays an obligatory role in regulating whole body metabolic health and therefore has been investigated in the context of maternal exercise. Stanford et al. recently determined maternal exercise improved glucose tolerance in mice born to mothers fed a standard chow or high-fat diet [120]. Interestingly, their ex vivo experiments revealed no effect of maternal exercise on the skeletal muscle, but instead a robust remodeling of the hepatic insulin sensitivity and glucose production phenotype. Although the mechanisms have yet to be elucidated, studies from the same group show evidence of hepatic mitochondrial biogenesis in the offspring born to mothers who exercised. Although attention has been centered on determining the effect of maternal obesity and gestational diabetes on offspring $\beta$-cell function [128131], Zheng et al. was the first to show that the combination of pregestational paternal exercise and pre-and during-gestational exercise preserved $\beta$-cell mass, size, and islet morphology in offspring born to parents fed a high-fat diet [132].

To summarize, maternal exercise has been shown to improve cardiovascular function, skeletal muscle oxidative capacity, and whole-body substrate metabolism partly as a result of tissue-specific improvements in skeletal muscle, liver, and pancreatic phenotypes. It is believed that epigenetic modifications underpin these improvements, specifically in genes that affect mitochondrial outcomes of these tissues. Unfortunately, most of these findings are derived from animal studies and thus, it is still unclear if these results can be translated to humans. These specific types of studies are wrought with challenges in human cohorts due to the invasiveness of tissue sampling procedures. Often, human studies are limited to the presence/absence of metabolites or hormones in cord blood and tissue. Thus, there is a need to identify new avenues for future studies that explore the transmission and signaling behind maternal and fetal health and disease in the context of human tissues and metabolism.

\section{Recommendations for Future Studies}

\section{Intrauterine microenvironment}

Previously, exercise has been shown to induce robust changes in circulating factors that affect the tissue's microenvironment. To date, the most studied exercise "factors" are the cytokines released from skeletal muscle, termed myokines. There are presently 600 skeletal muscle myokine species that have been identified [133], and targeted approaches aim to understand the effects of myokines in remodeling skeletal muscle metabolism. For example, interleukin 6, brain-derived neurotrophic factor, and interleukin 15 have been shown to 1) be secreted from skeletal muscle [134-136], 2) increase in circulation following exercise [134, 135, 137-139], and 3 ) independently improve mitochondrial density and/or function [140-142]. Importantly, these myokines help mediate the skeletal muscle-to-organ crosstalk and therefore may play a role in fetal programming resulting from maternal exercise, depending on their permeability through the placental-blood barrier. Recently, the novel myokine and adipokine known as apelin has been shown to mediate several of the skeletal muscle phenotype changes that occur in mice offspring born to mothers who exercised during pregnancy [87]. In this study, maternal exercise increased levels of apelin, which subsequently increases mitochondrial biogenesis and oxidative capacity in the offspring. Like apelin, other undiscovered myokines and/or adipokines, which are now termed "exerkines" in the field when they are secreted in response to exercise, may be signaling from mother to infant and lend support to the notion of maternal exercise improving offspring metabolic health.

\section{Maternal donation of mtDNA}

Mitochondria are originally descendent from endosymbiotic bacterium and this derivation from symbiotic ancestors allows the maintenance of their own genome (mtDNA) [143, 144]. mtDNA consists of a DNA ring of approximately 16570 nucleotides and contains 37 genes [145] but is responsible for transcription of 13 essential electron transport chain (ETC) proteins, 2 rRNAs, and 22 tRNAs [143]. The remainder of the nearly 1200 proteins that make up mitochondria require nuclear transcription and subsequent import into appropriate mitochondrial compartments resulting in a finely tuned coordination between mtDNA and nuclear DNA.

Unlike the nuclear genome, mtDNA is inherited strictly through a maternal inheritance pattern in eukaryotes where only the oocyte contributes mtDNA to the offspring [141]. Several mechanisms are recognized for the elimination of paternal mtDNA from the embryo and include a genetic bottleneck, autophagy post-fertilization, ubiquitin-protease pathways, and altered paternal mitochondrial transcription factor A (TFAM) expression [146]. In addition, while exclusive maternal donation of mitochondria and 
mtDNA is generally acknowledged, a few, exceptional cases of biparental inheritance of mtDNA in humans exist [147]. This lack of recombination of mtDNA and its unique inheritance pattern thus allows for an accumulation of transmitted mutations which can lead to severe diseases in the offspring.

When maternal obesity was studied across three generations of mice, its effects could be tracked across all three generations alongside mitochondrial changes in morphology, bioenergetics, and dynamics [116]. The first generation of female offspring (F1) showed peripheral insulin resistance, increased intramuscular lipid content, mitochondrial dysfunction, and impaired mitochondrial dynamics in skeletal muscle. Oocyte mitochondria from the F1 mice also showed deranged morphology, reduced mtDNA copy number, and impaired mitochondrial dynamics. These were also apparent in the subsequent two generations. This propagation of mitochondrial impairments across generations is not restricted to skeletal muscle. A follow-up study by this group showed that maternal obesity in mice results in transgenerational cardiac mitochondrial deficiencies as well [148]. Elegant in vitro fertilization studies from another group have shown that the oocyte at the time of fertilization is susceptible to the intrauterine environment [149]. Whether or not this is generating changes in mtDNA or some nuclear aspect remains to be discovered and is the central crux to understanding the inheritability of the mitochondrial phenotype. Nonetheless, the initial insult is presently thought to stem from changes in oocyte mitochondria, particularly issues with mitophagy in the oocyte [150], that propagate to mitochondria that are present in all tissues/organs. Finally, it is not certain in rodents or humans if exercise is protective in rescuing a deleterious mitochondrial phenotype that is seen in maternal obesity. With new evidence of transmission of mitochondrial impairment and as mtDNA codes for critical bioenergetic genes, any potent approaches of improving mitochondrial health could lead to substantial advancements of offspring health.

\section{Umbilical cord-derived mesenchymal stem cells}

Human trials examining the effects of maternal exercise on offspring have primarily measured body composition and epigenetic outcomes in placental biospecimens such as umbilical cord and cord blood. Although much has been gained from rodent models, several discrepancies exist including a gestational period that is significantly shorter than humans as well as differences in placental physiology including estrogen synthesis/release [151], miRNA profile [152], expression of cell surface markers for trophoblast invasion [153], and accumulation of diet-specific metabolites [154]. Thus, it remains unclear if rodent findings can be translated to humans.

Non-invasive means to examine the effects of maternal exercise on offspring skeletal muscle metabolism need to be implemented in humans. Primary human skeletal muscle cells (SKMcs) have been used to investigate cell-autonomous mechanisms that underly the effects of lifestyle interventions including exercise [155], as well as the pathophysiology of diseases including diabetes [156], obesity $[157,158]$, and peripheral arterial disease [148]. For example, SKMcs derived from exercise-trained subjects retain the hallmark adaptations seen in vivo including, elevations in lipid handling capacity $[159,160]$, oxidative capacity [155], and insulin sensitivity [161]. Thus, it is believed the phenotype expressed in vitro is the result of lasting metabolic programming which occurs in vivo. Therefore, it is plausible to suggest that the metabolic programming in SKMcs results from extrinsic changes in the tissue microenvironment, similar to what may be occurring in the intrauterine environment. Performing invasive measures such as muscle biopsies in young infants and children is impractical, making it difficult to understand the tissue-specific effects of maternal programming unique to exercise training. To test these hypotheses, researchers must identify a primary cell niche that can be noninvasively obtained and exists beyond the placental-blood barrier.

Recently, blood and umbilical cord-derived mesenchymal stem cells (MSCs) have gained attention in regenerative medicine because of their ability to differentiate into several cell types including chondrocytes, adipocytes, and skeletal myocytes [162]. Importantly, MSCs are of fetal origin and thus, a mesodermal stem cell lineage that contributes to the fetal development of several peripheral tissues and are the primary stem cell lineage responsible for fetal myogenesis as well as postnatal skeletal muscle growth and repair. Therefore, MSCs also offer the potential to gain insight into the metabolic phenotype of the developing skeletal muscle at birth and possibly how it will be maintained into adulthood. Recently, Boyle et al. indicated that MSCs from offspring born to obese mothers have lower rates of fat oxidation and elevated rates of lipid deposition [163]. These same MSCs were differentiated into an adipogenic phenotype and measured for the quantity of fat stored in these cells. Interestingly, this fat storage phenotype was shown to correlate positively with infant fat mass, which is direct support for translating this model to the phenotype of the infant. Interestingly, when stratified by oxidation rates, offspring with low MSC oxidation rates had higher adiposity and fasting plasma insulin levels in vivo, providing evidence that maternal obesity has lasting negative implications for the metabolic phenotype of the infant [164]. Although maternal physical activity has been shown to have a beneficial effect on rodent offspring, it has yet to be determined whether this is evident in humans and elicited primarily by the intrauterine environment. Thus, MSCs may bridge the gap for future investigations into this area and may be used as an in vitro model for myogenic outcomes but also for exploring the inheritance of the maternal mitochondrial phenotype.

\section{Conclusion}

The aim of the present review was to examine the current knowledge in the field of exercise in pregnancy as it relates to the mother and developing fetus and identify gaps in the literature (summarized in - Fig. 1). Animal studies have outlined several mechanisms through which the metabolic health of offspring is improved through maternal exercise and have established inheritance of metabolic impairments that track across multiple generations. Through these studies, mitochondria seem to be key organelles in the progression of metabolic health across generations. Exerkines may be a new research area of understanding how maternal exercise may signal changes to the developing fetus. In addition, MSCs present themselves as a potential and relevant model to gain insight into this cellular and metabolic programming of offspring. With these models, exercise scientists may soon have the necessary tools to 


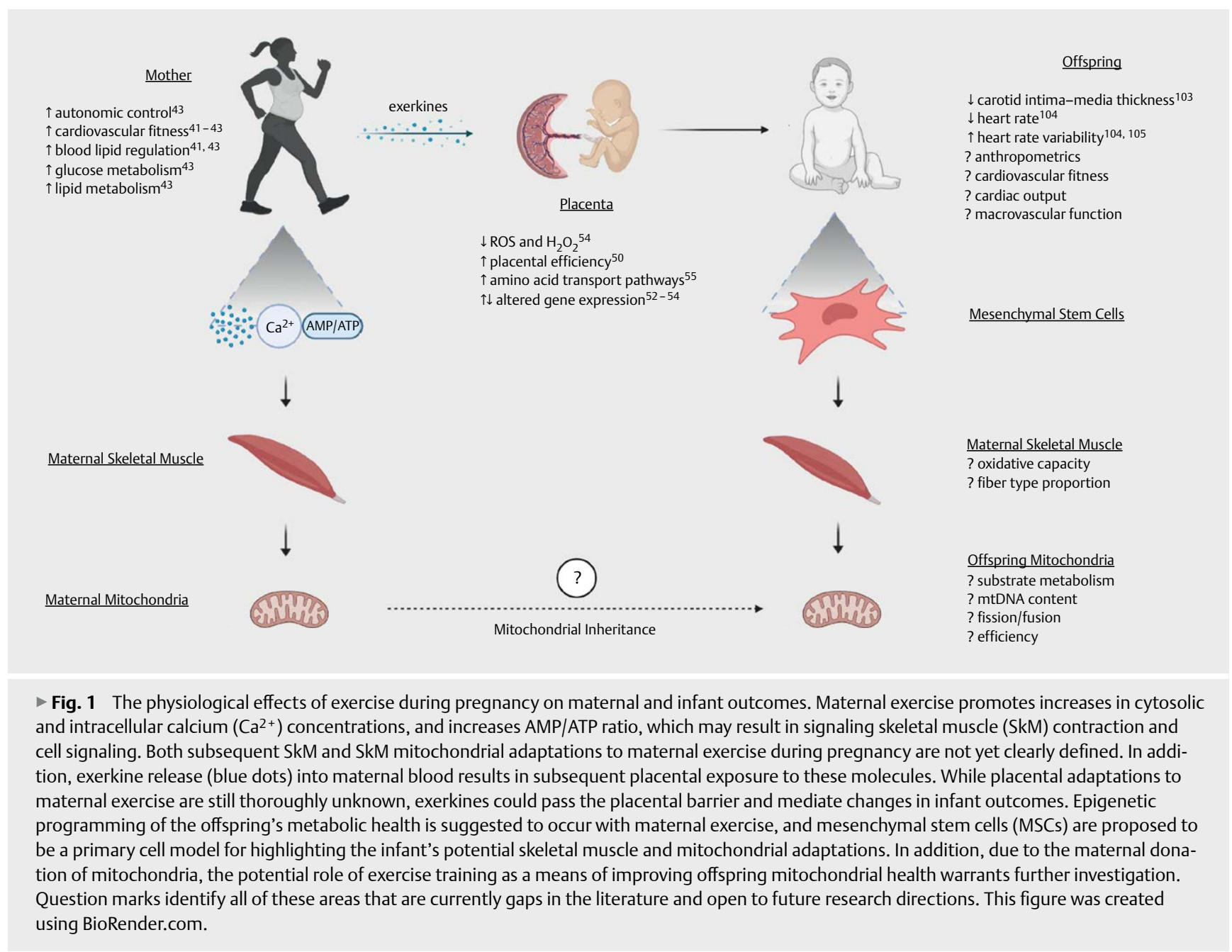

explore the advantageous mechanisms in humans that exercise elicits from mother to offspring. There is a dire need to translate these findings to human cohorts as exercise during pregnancy maybe be a viable nonpharmacological strategy for prevention of metabolic diseases at the earliest timepoint - while in the womb.

\section{Conflict of Interest}

The authors have nothing to disclose. The authors confirm that the manuscript was built based on the IJSM ethical standards [165].

\section{References}

[1] Guh DP, Zhang W, Bansback N et al. The incidence of co-morbidities related to obesity and overweight: A systematic review and meta-analysis. BMC Public Health 2009; 9: 88. doi:10.1186/14712458-9-88

[2] Nishijima $\mathrm{H}$, Satake $\mathrm{K}$, Igarashi $\mathrm{K}$ et al. Effects of exercise in overweight Japanese with multiple cardiovascular risk factors. Med Sci Sports Exerc 2007; 39: 926-933. doi:10.1249/ mss.0b013e3180383d84
[3] Donnelly JE, Honas J], Smith BK et al. Aerobic exercise alone results in clinically significant weight loss for men and women: Midwest exercise trial 2. Obesity (Silver Spring) 2013; 21: E219-E228. doi:10.1002/oby.20145

[4] Irwin ML, Yasui Y, Ulrich CM et al. Effect of Exercise on total and intra-abdominal body fat in postmenopausal women. JAMA 2003; 289: 323-330. doi:10.1001/jama.289.3.323

[5] Blumenthal JA, Sherwood A, Gullette ECD et al. Exercise and weight loss reduce blood pressure in men and women with mild hypertension. Arch Intern Med 2000; 160: 1947-1958

[6] Hellènius M-L, Faire Ud, Berglund B et al. Diet and exercise are equally effective in reducing risk for cardiovascular disease. Results of a randomized controlled study in men with slightly to mederately raised cardiovascular risk factors. Atherosclerosis 1993; 103: 81-91

[7] Posner JD, Gorman KM, Windsor-Landsberg L et al. Low to moderate intensity endurance training in healthy older adults: Physiological responses after four months. J Am Geriatr Soc 1992; 40: 1-7

[8] Bonanno JA, Lies JE. Effects of physical training on coronary risk factors. Am J Cardiol 1974; 33: 760-764

[9] DiPietro L, Seeman TE, Stachenfeld NS et al. Moderate-intensity aerobic training improves glucose tolerance in aging independent of abdominal adiposity. J Am Geriatr Soc 1998; 46: 875-879 
[10] Chang SH, Kim K, Lee J et al. The effectiveness of physical activity interventions for low-income and ethnic minority children and youths: A meta-analysis. J Phys Act Health 2019; 16: 799-808. doi:10.1123/jpah.2018-0648

[11] Jenum AK, Anderssen SA, Birkeland KI et al. Promoting physical activity in a low-income multiethnic district: effects of a community intervention study to reduce risk factors for type 2 diabetes and cardiovascular disease: a community intervention reducing inactivity. Diabetes Care 2006; 29: 1605-1612. doi:10.2337/dc05-1587

[12] [Anonym] Physical activity and exercise during pregnancy and the postpartum period: ACOG committee opinion, number 804 . Obstet Gynecol 2020; 135: e178-e188. doi:10.1097| aog. 0000000000003772

[13] Barker DJ, Winter PD, Osmond C et al. Weight in infancy and death from ischaemic heart disease. Lancet 1989; 2: 577-580. doi:10.1016/ s0140-6736(89)90710-1

[14] Bentov $\mathrm{Y}$, Yavorska T, Esfandiari N et al. The contribution of mitochondrial function to reproductive aging. J Assist Reprod Genet 2011; 28: 773-783. doi:10.1007/s10815-011-9588-7

[15] Catalano PM, Shankar K. Obesity and pregnancy: mechanisms of short term and long term adverse consequences for mother and child. BMJ 2017; 356: j1. doi:10.1136/bmj.j1

[16] Phelan S. Pregnancy: a "teachable moment" for weight control and obesity prevention. Am J Obstet Gynecol 2010; 202: 135 e131-e138. doi:10.1016/j.ajog.2009.06.008

[17] Wolfe LA, Weissgerber TL. Clinical physiology of exercise in pregnancy: A literature review. J Obstet Gynaecol Can 2003; 25: 473-483. doi:10.1016/s1701-2163(16)30309-7

[18] Barakat R, Perales M. Resistance exercise in pregnancy and outcome. Clin Obstet Gynecol 2016; 59: 591-599

[19] Piercy KL, Troiano RP, Ballard RM et al. The physical activity guidelines for Americans. JAMA 2018; 320: 2020-2028. doi:10.1001/ jama.2018.14854

[20] Garber CE, Blissmer B, Deschenes MR et al. American College of Sports Medicine position stand. Quantity and quality of exercise for developing and maintaining cardiorespiratory, musculoskeletal, and neuromotor fitness in apparently healthy adults: Guidance for prescribing exercise. Med Sci Sports Exerc 2011; 43: 1334-1359. doi:10.1249/MSS.0b013e318213fefb

[21] de Oliveria Melo AS, Silva JL, Tavares JS et al. Effect of a physical exercise program during pregnancy on uteroplacental and fetal blood flow and fetal growth: A randomized controlled trial. Obstet Gynecol 2012; 120: 302-310. doi:10.1097/AOG.0b013e31825de592

[22] Price BB, Amini SB, Kappeler K. Exercise in pregnancy: effect on fitness and obstetric outcomes-a randomized trial. Med Sci Sports Exerc 2012; 44: 2263-2269. doi:10.1249/MSS.0b013e318267ad67

[23] Owe KM, Nystad W, Skjaerven R et al. Exercise during pregnancy and the gestational age distribution: a cohort study. Med Sci Sports Exerc 2012; 44: 1067-1074. doi:10.1249/MSS.0b013e3182442fc9

[24] Barakat R, Pelaez M, Montejo R et al. Exercise throughout pregnancy does not cause preterm delivery: A randomized, controlled trial. J Phys Act Health 2014; 11: 1012-1017. doi:10.1123/jpah.2012-0344

[25] Zhang J, Savitz DA. Exercise during pregnancy among US women. Ann Epidemiol 1996; 6: 53-59. doi:10.1016/1047-2797(95)00093-3

[26] Nascimento SL, Surita FG, Godoy AC et al. Physical activity patterns and factors related to exercise during pregnancy: A cross sectional study. PLoS One 2015; 10: e0128953. doi:10.1371/journal. pone. 0128953

[27] Evenson KR, Savitz DA, Huston SL. Leisure-time physical activity among pregnant women in the US. Paediatr Perinat Epidemiol 2004; 18: $400-407$
[28] Walsh JM, McGowan C, Byrne J et al. Prevalence of physical activity among healthy pregnant women in Ireland. Int J Gynaecol Obstet 2011; 114: 154-155. doi:10.1016/j.ijgo.2011.02.016

[29] Hegaard HK, Damm P, Hedegaard M et al. Sports and leisure time physical activity during pregnancy in nulliparous women. Matern Child Health J 2011; 15: 806-813. doi:10.1007/s10995-010-0647-y

[30] Liu ], Blair SN, Teng Y et al. Physical activity during pregnancy in a prospective cohort of British women: results from the Avon longitudinal study of parents and children. Eur J Epidemiol 2011; 26: 237-247. doi:10.1007/s10654-010-9538-1

[31] Domingues MR, Barros AJD. Leisure-time physical activity during pregnancy in the 2004 Pelotas Birth Cohort Study. Rev Saude Publica 2007; 41: 173-180

[32] Nascimento SL, Surita FG, Cecatti JG. Physical exercise during pregnancy: a systematic review. Curr Opin Obstet Gynecol 2012; 24: 387-394. doi:10.1097/GCO.0b013e328359f131

[33] Callaway LK, Colditz PB, Byrne NM et al. Prevention of gestational diabetes: feasibility issues for an exercise intervention in obese pregnant women. Diabetes Care 2010; 33: 1457-1459. doi:10.2337| dc09-2336

[34] Peaceman AM, Clifton RG, Phelan S et al. Lifestyle interventions limit gestational weight gain in women with overweight or obesity: LIFE-Moms prospective meta-analysis. Obesity (Silver Spring) 2018; 26: 1396-1404. doi:10.1002/oby.22250

[35] Redman LM, Drews KL, Klein S et al. Attenuated early pregnancy weight gain by prenatal lifestyle interventions does not prevent gestational diabetes in the LIFE-Moms consortium. Diabetes Res Clin Pract 2021; 171: 108549. doi:10.1016/j.diabres.2020.108549

[36] May LE, Knowlton J, Hanson J et al. Effects of exercise during pregnancy on maternal heart rate and heart rate variability. PM R 2016; 8: 611-617. doi:10.1016/j.pmrj.2015.11.006

[37] Martin CL, Brunner Huber LR. Physical activity and hypertensive complications during pregnancy: Findings from 2004 to 2006 north carolina pregnancy risk assessment monitoring system. Birth 2010; 37: 202-210. doi:10.1111/j.1523-536x.2010.00407.x

[38] Kawabata I, Nakai A, Sekiguchi A et al. The effect of regular exercise training during pregnancy on postpartum brachial-ankle pulse wave velocity, a measure of arterial stiffness. J Sports Sci Med 2012; 11: 489-494

[39] Haakstad LAH, Edvardsen E, Bø K. Effect of regular exercise on blood pressure in normotensive pregnant women. A randomized controlled trial. Hypertens Pregnancy 2016; 35: 170-180. doi:10.3109/106419 55.2015.1122036

[40] Barakat R, Pelaez M, Cordero Y et al. Exercise during pregnancy protects against hypertension and macrosomia: randomized clinical trial. Am J Obstet Gynecol 2016; 214: 649.e1-649.e8. doi:10.1016/j. ajog.2015.11.039

[41] May LE, Allen J], Gustafson KM. Fetal and maternal cardiac responses to physical activity and exercise during pregnancy. Early Hum Dev 2016; 94: 49-52. doi:10.1016/j.earlhumdev.2016.01.005

[42] Mottola MF, Artal R. Fetal and maternal metabolic responses to exercise during pregnancy. Early Hum Dev 2016; 94: 33-41. doi:10.1016/j.earlhumdev.2016.01.008

[43] Newton ER, May L. Adaptation of maternal-fetal physiology to exercise in pregnancy: The basis of guidelines for physical activity in pregnancy. Clin Med Insights Womens Health 2017; 10: 1179562X17693224. doi:10.1177/1179562X17693224

[44] Hytten F, Chamberlain G. Clinical Physiology in Obstetrics. Oxford: Blackwell Scientific Publications; 1991

[45] Gabbe S, Neibyl J, Simpson J. Obstetrics: Normal and Problem Pregnancies. 2nd ed. New York: Churchill Livingstone; 1991 
[46] Mottola MF, Davenport MH, Brun CR et al. VO2peak prediction and exercise prescription for pregnant women. Med Sci Sports Exerc 2006; 38: 1389-1395. doi:10.1249/01.mss.0000228940.09411.9c

[47] Khodiguian N, Jaque-Fortunato SV, Wiswell RA et al. A comparison of cross-sectional and longitudinal methods of assessing the influence of pregnancy on cardiac function during exercise. Semin Perinatol 1996; 20: 232-241

[48] Clapp JF. The effects of maternal exercise on fetal oxygenation and feto-placental growth. Eur J Obstet Gynecol Reprod Biol 2003; 110: S80-S85. doi:10.1016/s0301-2115(03)00176-3

[49] Clapp JF 3rd.. Maternal carbohydrate intake and pregnancy outcome. Proc Nutr Soc 2002; 61: 45-50. doi:10.1079/pns2001129

[50] Reyes LM, Davenport MH. Exercise as a therapeutic intervention to optimize fetal weight. Pharmacol Res 2018; 132: 160-167. doi:10.1016/j.phrs.2018.04.016

[51] Rowell LB. Human cardiovascular adjustments to exercise and thermal stress. Physiol Rev 1974; 54: 75-159

[52] Jones MT, Norton KI, Dengel DR et al. Effects of training on reproductive tissue blood flow in exercising pregnant rats. J Appl Physiol (1985) 1990; 69: 2097-2103

[53] Pivarnik JM, Mauer MB, Ayres NA et al. Effects of chronic exercise on blood volume expansion and hematologic indices during pregnancy. Obstet Gynecol 1994; 83: 265-269

[54] Szymanski LM, Satin AJ. Strenuous exercise during pregnancy: is there a limit? Am J Obstet Gynecol 2012; 207: 179 e171-e176. doi:10.1016/j.ajog.2012.07.021

[55] Brett KE, Ferraro ZM, Holcik M et al. Prenatal physical activity and diet composition affect the expression of nutrient transporters and mTOR signaling molecules in the human placenta. Placenta 2015; 36: 204-212. doi:10.1016/j.placenta.2014.11.015

[56] Hutchinson KA, Vuong NH, Mohammad S et al. Physical activity during pregnancy is associated with increased placental FATP4 protein expression. Reprod Sci 2020; 27: 1909-1919. doi:10.1007| s43032-020-00210-w

[57] Ramirez-Velez R, Bustamante J, Czerniczyniec A et al. Effect of exercise training on eNOS expression, NO production and oxygen metabolism in human placenta. PLoS One 2013; 8: e80225. doi:10.1371/journal.pone.0080225

[58] Day PE, Ntani G, Crozier SR et al. Maternal factors are associated with the expression of placental genes involved in amino acid metabolism and transport. PLoS One 2015; 10: e0143653. doi:10.1371/journal. pone. 0143653

[59] Son JS, Liu X, Tian Q et al. Exercise prevents the adverse effects of maternal obesity on placental vascularization and fetal growth. J Physiol 2019; 597: 3333-3347. doi:10.1113/jp277698

[60] Song L, Yan J, Wang $N$ et al. Prenatal exercise reverses high-fat-dietinduced placental alterations and alters male fetal hypothalamus during late gestation in rats†. Biol Reprod 2019; 102: 705-716. doi:10.1093/biolre/ioz213

[61] Mangwiro YTM, Cuffe JSM, Mahizir D et al. Exercise initiated during pregnancy in rats born growth restricted alters placental mTOR and nutrient transporter expression. J Physiol 2019; 597: 1905-1918. doi:10.1113/jp277227

[62] Baird ], Fisher D, Lucas P et al. Being big or growing fast: systematic review of size and growth in infancy and later obesity. BMJ 2005; 331: 929. doi:10.1136/bmj.38586.411273.EO.

[63] Boney CM, Verma A, Tucker $R$ et al. Metabolic syndrome in childhood: association with birth weight, maternal obesity, and gestational diabetes mellitus. Pediatrics 2005; 115: e290-e296. doi:10.1542/peds.2004-1808
[64] Nordman H, Jääskeläinen J, Voutilainen R. Birth size as a determinant of cardiometabolic risk factors in children. Horm Res Paediatr 2020; 93: 144-153. doi:10.1159/000509932

[65] Pettitt D], Jovanovic L. Birth weight as a predictor of type 2 diabetes mellitus: The U-shaped curve. Curr Diab Rep 2001; 1: 78-81. doi:10.1007/s11892-001-0014-x

[66] Lumey LH, Khalangot MD, Vaiserman AM. Association between type 2 diabetes and prenatal exposure to the Ukraine famine of 1932-33: a retrospective cohort study. Lancet Diabetes Endocrinol 2015; 3: 787-794. doi:10.1016/s2213-8587(15)00279-x

[67] Lahti-Pulkkinen M, Bhattacharya S, Wild SH et al. Consequences of being overweight or obese during pregnancy on diabetes in the offspring: a record linkage study in Aberdeen, Scotland. Diabetologia 2019; 62: 1412-1419. doi:10.1007/s00125-019-4891-4

[68] Kaar JL, Crume T, Brinton JT et al. Maternal obesity, gestational weight gain, and offspring adiposity: The exploring perinatal outcomes among children study. J Pediatr 2014; 165: 509-515. doi:10.1016/j.jpeds.2014.05.050

[69] Jönsson J, Renault KM, García-Calzón S et al. Lifestyle intervention in pregnant women with obesity impacts cord blood dna methylation which associates with body composition in the offspring. Diabetes 2021; 70: 854-866. doi:10.2337/db20-0487

[70] Dahly DL, Li X, Smith HA et al. Associations between maternal lifestyle factors and neonatal body composition in the Screening for Pregnancy Endpoints (Cork) cohort study. Int J Epidemiol 2018; 47: 131-145. doi:10.1093/ije/dyx221

[71] Mudd LM, Pivarnik J, Holzman CB et al. Leisure-time physical activity in pregnancy and the birth weight distribution: Where is the effect? J Phys Act Health 2012; 9: 1168-1177

[72] Ferrari N, Bae-Gartz I, Bauer C et al. Exercise during pregnancy and its impact on mothers and offspring in humans and mice. J Dev Orig Health Dis 2018; 9: 63-76. doi:10.1017/\$2040174417000617

[73] Guillemette L, Hay JL, Kehler DS et al. Exercise in pregnancy and children's cardiometabolic risk factors: A systematic review and meta-analysis. Sports Med Open 2018; 4: 35. doi:10.1186/ s40798-018-0148-x

[74] Clapp JF. Influence of endurance exercise and diet on human placental development and fetal growth. Placenta 2006; 27: 527-534. doi:10.1016/j.placenta.2005.07.010

[75] Clapp JF 3rd, Capeless EL. Neonatal morphometrics after endurance exercise during pregnancy. Am J Obstet Gynecol 1990; 163: 1805-1811. doi:10.1016/0002-9378(90)90754-u

[76] Bisson M, Lavoie-Guénette J, Tremblay A et al. Physical activity volumes during pregnancy: A systematic review and meta-analysis of observational studies assessing the association with infant's birth weight. AJP Rep 2016; 06: e170-e197. doi:10.1055/s-0036-1583169

[77] Hegaard HK, Pedersen BK, Nielsen BB et al. Leisure time physical activity during pregnancy and impact on gestational diabetes mellitus, pre-eclampsia, preterm delivery and birth weight: a review. Acta Obstet Gynecol Scand 2007; 86: 1290-1296. doi:10.1080/00016340701647341

[78] Owe KM, Nystad W, Bø K. Association between regular exercise and excessive newborn birth weight. Obstet Gynecol 2009; 114: 770-776. doi:10.1097/AOG.0b013e3181b6c105

[79] Alderman BW, Zhao H, Holt VL et al. Maternal physical activity in pregnancy and infant size for gestational age. Ann Epidemiol 1998; 8: 513-519

[80] Juhl M, Olsen J, Andersen PK et al. Physical exercise during pregnancy and fetal growth measures: A study within the Danish National Birth Cohort. Am J Obstet Gynecol 2010; 202: 63.e1-63.e8. doi:10.1016/j. ajog.2009.07.033 
[81] Clapp JF 3rd. Long-term outcome after exercising throughout pregnancy: fitness and cardiovascular risk. Am J Obstet Gynecol 2008; 199: 489 e481-e486. doi:10.1016/j.ajog.2008.05.006

[82] Davenport MH, Sobierajski F, Mottola MF et al. Glucose responses to acute and chronic exercise during pregnancy: A systematic review and meta-analysis. Br J Sports Med 2018; 52: 1357-1366. doi:10.1136/bjsports-2018-099829

[83] Quiclet C, Dubouchaud H, Berthon P et al. Maternal exercise modifies body composition and energy substrates handling in male offspring fed a high-fat/high-sucrose diet. J Physiol 2017; 595: 7049-7062

[84] Carter LG, Lewis KN, Wilkerson DC et al. Perinatal exercise improves glucose homeostasis in adult offspring. Am J Physiol Endocrinol Metab 2012; 303: E1061-E1068. doi:10.1152/ajpendo.00213.2012

[85] Blaize AN, Breslin E, Donkin SS et al. Maternal exercise does not significantly alter adult rat offspring vascular function. Med Sci Sports Exerc 2015; 47: 2340-2346. doi:10.1249/mss.0000000000000665

[86] Fick A. Ueber die Messung des Blutquantum in den Herzventrikeln. Sb Phys Med Ges Worzburg 1870; 16-17

[87] Son JS, Chae SA, Wang $\mathrm{H}$ et al. Maternal inactivity programs skeletal muscle dysfunction in offspring mice by attenuating apelin signaling and mitochondrial biogenesis. Cell Rep 2020; 33: 108461

[88] Eclarinal JD, Zhu S, Baker MS et al. Maternal exercise during pregnancy promotes physical activity in adult offspring. FASEB J 2016; 30: 2541-2548

[89] Wilmore JH, Stanforth PR, Gagnon J et al. Cardiac output and stroke volume changes with endurance training: The HERITAGE Family Study. Med Sci Sports Exerc 2001; 33: 99-106

[90] Hambrecht R, Gielen S, Linke A et al. Effects of exercise training on left ventricular function and peripheral resistance in patients with chronic heart failure: A randomized trial. JAMA 2000; 283: 30953101

[91] Carter JB, Banister EW, Blaber AP. Effect of endurance exercise on autonomic control of heart rate. Sports Med 2003; 33: 33-46

[92] Martinmäki K, Häkkinen K, Mikkola J et al. Effect of low-dose endurance training on heart rate variability at rest and during an incremental maximal exercise test. Eur J Appl Physiol 2008; 104: 541-548

[93] Uusitalo AL, Laitinen T, Väisänen SB et al. Effects of endurance training on heart rate and blood pressure variability. Clin Physiol Funct Imaging 2002; 22: 173-179

[94] Harris JE, Pinckard KM, Wright KR et al. Exercise-induced 3'-sialyllactose in breast milk is a critical mediator to improve metabolic health and cardiac function in mouse offspring. Nat Metab 2020; 2 : 678-687

[95] Beeson JH, Blackmore HL, Carr SK et al. Maternal exercise intervention in obese pregnancy improves the cardiovascular health of the adult male offspring. Mol Metab 2018; 16: 35-44

[96] Chung E, Joiner HE, Skelton T et al. Maternal exercise upregulates mitochondrial gene expression and increases enzyme activity of fetal mouse hearts. Physiol Rep 2017; 5: e13184

[97] Currie KD, Thomas SG, Goodman JM. Effects of short-term endurance exercise training on vascular function in young males. Eur J Appl Physiol 2009; 107: 211-218

[98] Sugawara J, Komine $H$, Hayashi K et al. Reduction in $\alpha$-adrenergic receptor-mediated vascular tone contributes to improved arterial compliance with endurance training. Int J Cardiol 2009; 135: 346-352

[99] Hernandez JP, Franke WD. Effects of a 6-mo endurance-training program on venous compliance and maximal lower body negative pressure in older men and women. J Appl Physiol (1985) 2005; 99: 1070-1077
[100] Newcomer S, Taheripour P, Bahls M et al. Impact of porcine maternal aerobic exercise training during pregnancy on endothelial cell function of offspring at birth. J Dev Orig Health Dis 2012; 3: 04-09

[101] Bahls M, Sheldon RD, Taheripour $P$ et al. Mother's exercise during pregnancy programmes vasomotor function in adult offspring. Exp Physiol 2014; 99: 205-219

[102] Blaize N, Zartman E, Biel T et al. Impact of maternal exercise during pregnancy on arteiral function and atherosclerosis formation in swine offspring fed a high fat diet. Enliven: Gynecol Obestet 2015; 1: 005-015

[103] Blaize AN, Breslin E, Donkin SS et al. Maternal exercise does not significantly alter adult rat offspring vascular function. Med Sci Sports Exerc 2015; 47: 2340-2346

[104] Boonpattrawong NP, Golbidi S, Tai DC et al. Exercise during pregnancy mitigates the adverse effects of maternal obesity on adult male offspring vascular function and alters one-carbon metabolism. Physiol Rep 2020; 8: e14582

[105] Li S, Chen Y, Zhang Y et al. Exercise during pregnancy enhances vascular function via epigenetic repression of CaV1. 2 channel in offspring of hypertensive rats. Life Sci 2019; 231: 116576

[106] Brislane Á, Jones $\mathrm{H}$, Holder SM et al. The effect of exercise during pregnancy on maternal and offspring vascular outcomes: A pilot study. Reprod Sci 2020; 1-14

[107] May LE, Glaros A, Yeh H-W et al. Aerobic exercise during pregnancy influences fetal cardiac autonomic control of heart rate and heart rate variability. Early Hum Dev 2010; 86: 213-217

[108] May LE, Scholtz SA, Suminski R et al. Aerobic exercise during pregnancy influences infant heart rate variability at one month of age. Early Hum Dev 2014; 90: 33-38

[109] Ingjer F. Capillary supply and mitochondrial content of different skeletal muscle fiber types in untrained and endurance-trained men. A histochemical and ultrastructural study. Eur ] Appl Physiol Occup Physiol 1979; 40: 197-209

[110] Fritzen AM, Andersen SP, Qadri KAN et al. Effect of aerobic exercise training and deconditioning on oxidative capacity and muscle mitochondrial enzyme machinery in young and elderly individuals. J Clin Med 2020; 9: 3113

[111] Ingjer F. Effects of endurance training on muscle fibre ATP-ase activity, capillary supply and mitochondrial content in man. J Physiol 1979; 294: 419-432

[112] Liu J, Lee I, Feng $\mathrm{H}-\mathrm{Z}$ et al. Aerobic exercise preconception and during pregnancy enhances oxidative capacity in the hindlimb muscles of mice offspring. J Strength Cond Res 2018; 32: 1391-1403

[113] Siti F, Dubouchaud H, Hininger I et al. Maternal exercise before and during gestation modifies liver and muscle mitochondria in rat offspring. J Exp Biol 2019; 222: jeb194969

[114] Spiegelman BM. Transcriptional control of mitochondrial energy metabolism through the PGC1 coactivators. Novartis Found Symp 2007; 287: 60-63

[115] Laker RC, Lillard TS, Okutsu M et al. Exercise prevents maternal high-fat diet-induced hypermethylation of the Pgc- $1 \alpha$ gene and age-dependent metabolic dysfunction in the offspring. Diabetes 2014; 63: 1605-1611

[116] Huang T-Y, Zheng D, Houmard JA et al. Overexpression of PGC-1a increases peroxisomal biogenesis and mitochondrial lipid oxidation in human primary myotubes. FASEB J 2016; 30: 1246.1243-1246.1243

[117] Geng T, Li P, Okutsu M et al. PGC-1 $\alpha$ plays a functional role in exercise-induced mitochondrial biogenesis and angiogenesis but not fiber-type transformation in mouse skeletal muscle. Am J Physiol Cell Physiol 2010; 298: C572-C579

[118] Shulman GI. Ectopic fat in insulin resistance, dyslipidemia, and cardiometabolic disease. N Engl J Med 2014; 371: 1131-1141. doi:10.1056/NEJMra1011035 
[119] Saben J, Lindsey F, Zhong Y et al. Maternal obesity is associated with a lipotoxic placental environment. Placenta 2014; 35: 171-177. doi:10.1016/j.placenta.2014.01.003

[120] Raipuria M, Bahari H, Morris M]. Effects of maternal diet and exercise during pregnancy on glucose metabolism in skeletal muscle and fat of weanling rats. PLoS One 2015; 10: e0120980

[121] Stanford KI, Lee M-Y, Getchell KM et al. Exercise before and during pregnancy prevents the deleterious effects of maternal high-fat feeding on metabolic health of male offspring. Diabetes 2015; 64: 427-433

[122] Kasch J, Kanzleiter I, Saussenthaler $S$ et al. Insulin sensitivity linked skeletal muscle Nr4a1 DNA methylation is programmed by the maternal diet and modulated by voluntary exercise in mice. J Nutr Biochem 2018; 57: 86-92

[123] Stanford KI, Takahashi H, So K et al. Maternal exercise improves glucose tolerance in female offspring. Diabetes 2017; 66: 2124-2136

[124] Vega CC, Reyes-Castro LA, Bautista C] et al. Exercise in obese female rats has beneficial effects on maternal and male and female offspring metabolism. Int J Obes (Lond) 2015; 39: 712-719

[125] Zhou L, Xiao X, Li M et al. Maternal exercise improves high-fat diet-induced metabolic abnormalities and gut microbiota profiles in mouse dams and offspring. Front Cell Infect Microbiol 2020; 10: 292

[126] DeFronzo RA, Tripathy D. Skeletal muscle insulin resistance is the primary defect in type 2 diabetes. Diabetes Care 2009; 32: S157-S163. doi:10.2337/dc09-S302

[127] Carter LG, Qi NR, De Cabo R et al. Maternal exercise improves insulin sensitivity in mature rat offspring. Med Sci Sports Exerc 2013; 45: 832-840

[128] Dabelea D, Mayer-Davis E], Lamichhane AP et al. Association of intrauterine exposure to maternal diabetes and obesity with type 2 diabetes in youth: the SEARCH Case-Control Study. Diabetes care 2008; 31: 1422-1426

[129] Zambrano E, Sosa-Larios T, Calzada L et al. Decreased basal insulin secretion from pancreatic islets of pups in a rat model of maternal obesity. J Endocrinol 2016; 231: 49-57

[130] Nicholas LM, Nagao M, Kusinski LC et al. Exposure to maternal obesity programs sex differences in pancreatic islets of the offspring in mice. Diabetologia 2020; 63: 324-337

[131] Han J, Xu J, Epstein P et al. Long-term effect of maternal obesity on pancreatic beta cells of offspring: reduced beta cell adaptation to high glucose and high-fat diet challenges in adult female mouse offspring. Diabetologia 2005; 48: 1810-1818

[132] Zheng J, Alves-Wagner AB, Stanford KI et al. Maternal and paternal exercise regulate offspring metabolic health and beta cell phenotype. BMJ Open Diabetes Res Care 2020; 8: e000890

[133] Gorgens SW, Eckardt K, Jensen J et al. Exercise and regulation of adipokine and myokine production. Prog Mol Biol Transl Sci 2015; 135: 313-336. doi:10.1016/bs.pmbts.2015.07.002

[134] Keller P, Keller C, Carey AL et al. Interleukin-6 production by contracting human skeletal muscle: Autocrine regulation by IL-6. Biochem Biophys Res Commun 2003; 310: 550-554. doi:10.1016/j. bbrc.2003.09.048

[135] Cuppini R, Sartini S, Agostini D et al. BDNF expression in rat skeletal muscle after acute or repeated exercise. Arch Ital Biol 2007; 145: 99-110

[136] Nielsen AR, Mounier R, Plomgaard P et al. Expression of interleukin-15 in human skeletal muscle effect of exercise and muscle fibre type composition. J Physiol 2007; 584: 305-312. doi:10.1113/ jphysiol.2007.139618

[137] Pedersen BK, Akerstrom TC, Nielsen AR et al. Role of myokines in exercise and metabolism. J Appl Physiol (1985) 2007; 103: 1093-1098. doi:10.1152/japplphysiol.00080.2007
[138] Ogborn DI, Gardiner PF. Effects of exercise and muscle type on BDNF, NT-4/5, and TrKB expression in skeletal muscle. Muscle Nerve 2010; 41: 385-391. doi:10.1002/mus.21503

[139] Tamura Y, Watanabe K, Kantani T et al. Upregulation of circulating IL-15 by treadmill running in healthy individuals: Is IL-15 an endocrine mediator of the beneficial effects of endurance exercise? Endocr J 2011; 58: 211-215

[140] Wolsk E, Mygind H, Grondahl TS et al. IL-6 selectively stimulates fat metabolism in human skeletal muscle. Am J Physiol Endocrinol Metab 2010; 299: E832-E840. doi:10.1152/ajpendo.00328.2010

[141] Wood J, Tse MCL, Yang X et al. BDNF mimetic alleviates body weight gain in obese mice by enhancing mitochondrial biogenesis in skeletal muscle. Metabolism 2018; 87: 113-122. doi:10.1016/j.metabol.2018.06.007

[142] Yang X, Brobst D, Chan WS et al. Muscle-generated BDNF is a sexually dimorphic myokine that controls metabolic flexibility. Sci Signal 2019; 12: eaau1468. doi:10.1126/scisignal.aau1468

[143] Andersson S, Zomorodipour A, Andersson J et al. The genome sequence of Rickettsia prowazekii and the origin of the mitochondria. Nature 1998; 396: 133-140

[144] Calvo SE, Clauser KR, Mootha VK. MitoCarta2.0: an updated inventory of mammalian mitochondrial proteins. Nucleic Acids Res 2016; 44: D1251-D1257. doi:10.1093/nar/gkv1003

[145] Taanman J-W. The mitochondrial genome: Structure, transcription, translation and replication. Biochim Biophys Acta 1999; 1410: 103-123

[146] Zou W, Slone J, Cao Y et al. Mitochondria and their role in human reproduction. DNA Cell Biol 2020; 39: 1370-1378. doi:10.1089/ dna.2019.4807

[147] Luo S, Valencia CA, Zhang J et al. Biparental inheritance of mitochondrial DNA in humans. Proc Natl Acad Sci USA 2018; 115: 1303913044. doi:10.1073/pnas. 1810946115

[148] Ryan TE, Yamaguchi DJ, Schmidt CA et al. Extensive skeletal muscle cell mitochondriopathy distinguishes critical limb ischemia patients from claudicants. JCl Insight 2018; 3: e123235. doi:10.1172/jci. insight. 123235

[149] Wu LL, Russell DL, Wong SL et al. Mitochondrial dysfunction in oocytes of obese mothers: transmission to offspring and reversal by pharmacological endoplasmic reticulum stress inhibitors. Development 2015; 142: 681-691. doi:10.1242/dev.114850

[150] Boudoures AL, Saben J, Drury A et al. Obesity-exposed oocytes accumulate and transmit damaged mitochondria due to an inability to activate mitophagy. Dev Biol 2017; 426: 126-138. doi:10.1016/j. ydbio.2017.04.005

[151] Witorsch RJ. Low-dose in utero effects of xenoestrogens in mice and their relevance to humans: An analytical review of the literature. Food Chem Toxicol 2002; 40: 905-912

[152] Morales-Prieto DM, Chaiwangyen W, Ospina-Prieto S et al. MicroRNA expression profiles of trophoblastic cells. Placenta 2012; 33: 725-734. doi:10.1016/j.placenta.2012.05.009

[153] Soni C, Karande AA. Glycodelin A suppresses the cytolytic activity of CD8 + T lymphocytes. Mol Immunol 2010; 47: 2458-2466. doi:10.1016/j.molimm.2010.06.008

[154] Springer SA, Diaz SL, Gagneux P. Parallel evolution of a self-signal: humans and new world monkeys independently lost the cell surface sugar Neu5Gc. Immunogenetics 2014; 66: 671-674. doi:10.1007| s00251-014-0795-0

[155] Heden TD, Ryan TE, Ferrara PJ et al. Greater oxidative capacity in primary myotubes from endurance-trained women. Med Sci Sports Exerc 2017; 49: 2151-2157. doi:10.1249/MSS.0000000000001352 
[156] McAinch A], Steinberg GR, Mollica J et al. Differential regulation of adiponectin receptor gene expression by adiponectin and leptin in myotubes derived from obese and diabetic individuals. Obesity 2006; 14: 1898-1904

[157] Zou K, Hinkley JM, Park S et al. Altered tricarboxylic acid cycle flux in primary myotubes from severely obese humans. Int J Obes (Lond) 2019; 43: 895-905. doi:10.1038/s41366-018-0137-7

[158] Park S, Turner KD, Zheng D et al. Electrical pulse stimulation induces differential responses in insulin action in myotubes from severely obese individuals. J Physiol 2019; 597: 449-466. doi:10.1113/ JP276990

[159] Lund J, Helle SA, Li Y et al. Higher lipid turnover and oxidation in cultured human myotubes from athletic versus sedentary young male subjects. Sci Rep 2018; 8: 17549. doi:10.1038/s41598-01835715-7

[160] Lund J, Rustan AC, Lovsletten NG et al. Exercise in vivo marks human myotubes in vitro: Training-induced increase in lipid metabolism. PLoS One 2017; 12: e0175441. doi:10.1371/journal.pone.0175441
[161] Bourlier V, Saint-Laurent C, Louche K et al. Enhanced glucose metabolism is preserved in cultured primary myotubes from obese donors in response to exercise training. I Clin Endocrinol Metab 2013; 98: 3739-3747. doi:10.1210/jc.2013-1727

[162] Pittenger MF, Mackay AM, Beck SC et al. Multilineage potential of adult human mesenchymal stem cells. Science 1999; 284: 143-147

[163] Boyle KE, Patinkin ZW, Shapiro AL et al. Mesenchymal stem cells from infants born to obese mothers exhibit greater potential for adipogenesis: The Healthy Start BabyBUMP Project. Diabetes 2016; 65: 647-659. doi:10.2337/db15-0849

[164] Boyle KE, Patinkin ZW, Shapiro ALB et al. Maternal obesity alters fatty acid oxidation, AMPK activity, and associated DNA methylation in mesenchymal stem cells from human infants. Mol Metab 2017; 6: 1503-1516. doi:10.1016/j.molmet.2017.08.012

[165] Harriss D], MacSween A, Atkinson G. Ethical standards in sport and exercise science research: 2020 update. Int J Sports Med 2019; 40: 813-817. doi:10.1055/a-1015-3123 